\title{
Applicability of Different Estimated Glomerular Filtration Rate Equations in Chinese Healthy Population: A Cross-Sectional Study
}

\author{
Lu Wei \\ The First Affiliated Hospital of Nanjing Medical University \\ Xue Shen \\ The First Affiliated Hospital of Nanjing Medical University \\ Juan Zhang \\ The First Affiliated Hospital of Nanjing Medical University \\ Zhenzhu Yong \\ The First Affiliated Hospital of Nanjing Medical University \\ Qun Zhang \\ The First Affiliated Hospital of Nanjing Medical University \\ Weihong Zhao ( $\nabla$ zhaoweihongny@njmu.edu.cn ) \\ The First Affiliated Hospital of Nanjing Medical University
}

\section{Research Article}

Keywords: Glomerular filtration rate, equation, healthy, aging

Posted Date: June 11th, 2021

DOI: https://doi.org/10.21203/rs.3.rs-601868/v1

License: @ (1) This work is licensed under a Creative Commons Attribution 4.0 International License. Read Full License 


\section{Abstract}

Objective: To estimate kidney function with aging by equations based on serum creatinine (Scr) and investigate the applicability of these equations in estimated glomerular filtration rate (eGFR) in healthy Chinese population.

Patients and methods: A total of 34,416 healthy individuals were enrolled in this study, eGFR was calculated by CKD-EPI, FAS and Xiangya equations. Plotted scatter diagrams were adopted to show the difference between eGFR by above three equations. Agreement analysis between these equations was quantified by the linear weighted $\kappa$ statistic.

Results: eGFRs by CKD-EPI and Xiangya equation had a constant decline with aging. eGFR by FAS equation was approximately 116.5 $\mathrm{mL} / \mathrm{min} / 1.73 \mathrm{~m}^{2}$ under 40 years, then decreased with a rate of $1.27 \mathrm{~mL} / \mathrm{min} / 1.73 \mathrm{~m}^{2} /$ year. The difference was relatively small, and substantial agreement was observed between CKD-EPI and FAS equations with Scr levels above $0.9 \mathrm{mg} / \mathrm{dL}$ in males $(\kappa=0.798)$ or $0.7 \mathrm{mg} / \mathrm{dL}$ in females $(\kappa$ $=0.745)$. The agreement between CKD-EPI and FAS equations was moderate to near-perfect in non-elderly $(\kappa 0.617-0.901$ for males and 0.536 0.861 for females), whereas was fair to moderate in older adults ( $\kappa$ 0.494-0.513 for males and 0.373-0.526 for females). eGFR by CKD-EPI was slightly higher than which by FAS equation in older population. The agreement between Xiangya and CKD-EPI or FAS equation was all slight $(\kappa \square$ $0.2)$.

Conclusion: FAS equation could be used to evaluate declining rate of eGFR in healthy population. We recommend FAS or CKD-EPI equation in non-elderly adults, and FAS equation in older adults to calculate eGFR. More verification is needed, especially in older adults and population with low Scr level.

\section{Background}

Kidney function decline with aging, mainly reflected in the decline of glomerular filtration rate (GFR), following with structural and physiological changes [1]. With aging, kidney is more susceptible to acute kidney injury (AKI) as a result of reduced adaptive capacity [2]. Studies have shown that the risk of decreased kidney function increased by $74 \%$ per decade [3]. Thus, it is crucial to accurately evaluate kidney function and screen kidney disease in the physical examination population.

The accepted reference method for the determination of GFR is to use exogenous filtration markers or clearance techniques, such as clearance of inulin and dual-plasma sample clearance method [4]. These methods are accurate but invasive and unrealistic, thus they could not be widely implied in the physical examination population. Serum creatinine as estimates of GFR is inaccuracy[5]. As a result of these limitations, several equations have developed to evaluate estimated GFR (eGFR) [6-9].

The chronic kidney disease epidemiology collaboration (CKD-EPI) equation based on Scr was recommended by the 2012 Kidney Disease Improving Global Outcomes (KDIGO) guidelines for assessing eGFR [8]. Due to the relatively small sample of Asians, there is some limitation when used CKD-EPI equation to calculate eGFR in Asian population. In 2019, Li DY et al. developed Xiangya equation in a multi-ethnic Chinese population [7]. In the development of above equations, the proportion of healthy people was relatively few. Pottel et al. developed a full-agespectrum (FAS) equation based on 6,870 European healthy subjects in 2016, which had continuity throughout the age spectrum, avoided conversion for estimation equations between different age groups [6]. Validation studies have examined the performance of above equations in the Chinese population with chronic kidney disease [10-13], however, little is known about their evaluation for kidney function with aging in large healthy population.

In this study, we compare the CKD-EPI, FAS and Xiangya equation, and evaluate the performance of equations in healthy Chinese individuals by analyzing discrepancies between equations.

\section{Methods}

\section{Participants}

We collected the medical information on adults ( $\geq 18$ years) who underwent check-ups at the Health Management Center of The First Affiliated Hospital of Nanjing Medical University from January 2018 to January 2020. This study was approved by the ethics committee of the First Affiliated Hospital of Nanjing Medical University (approval No. 2018-SR-181). A rigorous filtering process was described in Fig. 1.

FAH-NMU: the First Affiliated Hospital of Nanjing Medical University, BMI: body mass index.

The inclusion criterion were as follows: (1) BMl:18.5-28.0 kg/m²; (2) No history of hypertension and Blood Pressure (BP) < 140/90 mmHg; (3) No history of diabetes, fasting blood glucose (FBG) $<7.0 \mathrm{mmol} / \mathrm{L}$; (4) No history of kidney disease, no proteinuria or hematuria; (5) No history of cardio-cerebrovascular disease, malignant tumor, thyroid disorders and systemic disease; (6) Serum uric acid (UA) < 420 umol/L for males or UA < $360 \mathrm{umol} / \mathrm{L}$ for females; alanine aminotransferase (ALT), low density lipoprotein cholesterol (LDL-C), high density lipoprotein cholesterol (HDL-C), 
total cholesterol (TC) were in normal range according to guideline based Chinese. Exclusion criterion included: (1) Lack of Scr value; (2) Pregnant women.

\section{Laboratory Measures}

Basic demographic information was collected using a physician-administered questionnaire. The questionnaire was evaluated for incomplete or inconsistent answers, by nurses and our staff physicians. Body mass index (BMI) was calculated as measured weight (kg) divided by height ( $\mathrm{m}$ ) squared.

All the fasting blood samples were assayed on AU5800 automatic analyzer (Beckman Coulter, Inc., USA), in strict accordance with the instructions of the apparatus. Scr was determined using the enzymatic method (Shanghai Kehua Dongling Diagnostic Products Co., Ltd, China) with a reference range 44-133umol/L. eGFR was calculated by CKD-EPI, FAS and Xiangya equations, which were shown in Table S1.

\section{Statistical Analyses}

All participants were divided into six subgroups according to age: $18-29$ years, 30-39 years, 40-49 years, 50-59 years, 60-69 years and $\geq 70$ years. Continuous variables were represented as mean \pm standard deviation (SD), categorical variables were represented as N/\%. Welch's ANOVA test was used to compare differences of variables between six subgroups, differences between males and females were tested with the independent-samples t test, $\mathrm{p}<0.05$ were considered statistically significant. We used partial correlation analysis to evaluate the relationship between the relevant factors and the differences of the three equations, and plotted scatter diagrams to directly reflect the relationship under different Scr and age levels. Agreement analysis between different equations was quantified using the linear weighted $k$ statistic. The criteria were as follows: $0 \leq \kappa$ value $<0.21$, slight agreement; $0.21 \leq \kappa$ value $<0.41$, fair agreement; $0.41 \leq \kappa$ value $<0.61$, moderate agreement; $0.61 \leq \kappa$ value $<0.81$, substantial agreement; and $0.81 \leq \kappa$ value $\leq 1.00$, near-perfect agreement. Statistical analyses were performed using the SPSS software version 25.0 (IBM Inc., USA) and MedCalc for Windows (version 19.6.1.0; MedCalc Software, Mariekerke, Belgium).

\section{Results}

\section{Characteristics of subjects}

A total of 34,416 healthy people were enrolled in this study. The mean age of total population was 39.0 years and $45.23 \%$ of which were males ( $\mathrm{N}$ = 15568). The mean Scr was $0.75 \mathrm{mg} / \mathrm{dL}$, the level of it showed stable before 40 years, then increased with aging. Other observation indices were shown in Table 1. 
Table 1

Baseline characteristics of the healthy subjects.

\begin{tabular}{|c|c|c|c|c|c|c|c|}
\hline \multirow[t]{2}{*}{ Variable } & All subjects & 18-29years & 30-39years & 40-49years & $50-59$ years & 60-69years & $\geq 70$ years \\
\hline & $(n=34416)$ & $(n=7226)$ & $(n=13358)$ & $(n=7890)$ & $(n=4046)$ & $(n=1413)$ & $(n=483)$ \\
\hline male $[\mathrm{n}(\%)]$ & $15568(45.23 \%)$ & $39.84(38.75 \%)$ & $5261(39.38 \%)$ & $3808(48.26 \%)$ & $2370(58.58 \%)$ & $906(64.12 \%)$ & $344(71.22 \%)$ \\
\hline $\mathrm{SBP}(\mathrm{mmHg})$ & $116.41(11.47)$ & $116.21(11.23)$ & $114.63(11.29)^{*}$ & $116.55(11.48)^{*}$ & $119.34(10.95)^{*}$ & $122.52(10.76)^{*}$ & $126.99(9.50)^{*}$ \\
\hline $\mathrm{DBP}(\mathrm{mmHg})$ & 71.58(8.34) & 70.72(7.97) & $70.74(8.18)$ & $72.30(8.61)^{*}$ & $73.88(8.45)^{*}$ & 73.98(8.08) & $70.76(8.00)^{*}$ \\
\hline $\mathrm{BMI}\left(\mathrm{kg} / \mathrm{m}^{2}\right)$ & $22.55(2.24)$ & $21.94(2.21)$ & $22.39(2.22)^{*}$ & $22.94(2.19)^{*}$ & $23.15(2.12)^{*}$ & $23.28(2.18)$ & $23.24(2.22)$ \\
\hline $\mathrm{HDL}(\mathrm{mmol} / \mathrm{L})$ & $1.44(0.27)$ & $1.45(0.26)$ & $1.44(0.27)$ & $1.45(0.28)$ & $1.44(0.28)$ & $1.43(0.27)$ & $1.43(0.28)$ \\
\hline $\begin{array}{l}\mathrm{LDL} \\
(\mathrm{mmol} / \mathrm{L})\end{array}$ & $2.95(0.53)$ & $2.81(0.53)$ & $2.91(0.52)^{*}$ & $3.03(0.51)^{*}$ & $3.13(0.50)^{*}$ & $3.08(0.54)^{*}$ & $2.91(0.62)^{*}$ \\
\hline $\mathrm{TG}(\mathrm{mmol} / \mathrm{L})$ & $1.07(0.40)$ & $0.95(0.36)$ & $1.04(0.39)^{*}$ & $1.12(0.41)^{*}$ & $1.20(0.41)^{\star}$ & $1.25(0.42)^{\star}$ & $1.17(0.34)^{*}$ \\
\hline $\mathrm{TC}(\mathrm{mmol} / \mathrm{L})$ & $4.88(0.70)$ & $4.68(0.70)$ & $4.82(0.69)^{*}$ & $5.00(0.66)^{*}$ & $5.11(0.64)^{*}$ & $5.09(0.70)$ & $4.88(0.80)^{\star}$ \\
\hline $\mathrm{FBG}(\mathrm{mmol} / \mathrm{L})$ & $5.07(0.46)$ & $4.94(0.41)$ & $5.02(0.41)^{*}$ & $5.13(0.46)^{*}$ & $5.24(0.51)^{\star}$ & $5.37(0.54)^{*}$ & $5.46(0.56)^{\star}$ \\
\hline $\operatorname{ALB}(\mathrm{g} / \mathrm{L})$ & $45.40(2.52)$ & $46.47(2.58)$ & $45.72(2.43)^{\star}$ & $44.88(2.34)^{*}$ & $44.60(2.33)^{*}$ & $44.12(2.13)^{*}$ & $42.93(2.35)^{*}$ \\
\hline UA(umol/L) & $297.27(60.92)$ & $298.10(60.61)$ & $292.27(61.05)^{\star}$ & $295.26(62.06)^{*}$ & $308.66(57.18)^{*}$ & $312.09(57.62)$ & 317.34(59.16) \\
\hline $\mathrm{BUN}(\mathrm{mmol} / \mathrm{L})$ & $4.76(1.12)$ & $4.55(1.08)$ & $4.65(1.07)^{\star}$ & $4.82(1.11)^{*}$ & $5.16(1.14)^{*}$ & $5.25(1.15)$ & $5.43(1.34)$ \\
\hline $\operatorname{Scr}(\mathrm{mg} / \mathrm{dL})$ & $0.75(0.15)$ & $0.73(0.15)$ & $0.73(0.15)$ & $0.75(0.15)^{*}$ & $0.79(0.15)^{*}$ & $0.80(0.14)^{*}$ & $0.84(0.16)^{*}$ \\
\hline
\end{tabular}

Values were presented as mean (standard deviation) or percent

Abbreviations: $S B P$ systolic blood pressure; $D B P$ diastolic blood pressure; $B M /$ body mass index; $H D L-C$ high density lipoprotein cholesterol; $L D L-C$ low-density lipoprotein cholesterol; $T G$ triglyceride; $T C$ total cholesterol; $F B G$ : fasting blood glucose; $A L B$ albumin; $U A$ uric acid; $B U N$ blood urea nitrogen.

${ }^{*} P<0.05$, compared with former age spectrum.

\section{Age-related trend of eGFR}

eGFRs calculated by three equations yielded a significant decline with aging. The tendency of eGFR calculated by CKD-EPI equations with aging showed the constant decline from $119.6 \mathrm{~mL} / \mathrm{min} / 1.73 \mathrm{~m}^{2}$ in $18-29$ years to $80.7 \mathrm{~mL} / \mathrm{min} / 1.73 \mathrm{~m}^{2}$ in above 70 years, and the decline rate of eGFR was $0.81 \mathrm{~mL} / \mathrm{min} / 1.73 \mathrm{~m}^{2} /$ year. The eGFR level by FAS equation was approximately stable with $116.5 \mathrm{~mL} / \mathrm{min} / 1.73 \mathrm{~m}{ }^{2}$ below age 40 , and gradually declined with a rate of $1.27 \mathrm{~mL} / \mathrm{min} / 1.73 \mathrm{~m}^{2} /$ year above age 40 with aging. Xiangya equations showed the constant decline from 98.1 to $73.7 \mathrm{~mL} / \mathrm{min} / 1.73 \mathrm{~m}^{2}$, the rate of decline was $0.56 \mathrm{~mL} / \mathrm{min} / 1.73 \mathrm{~m}^{2} /$ year. Descending trends of eGFR in subgroups by gender were shown in Fig. 2. Both males and females had similar descending trend as total population. Compared with males, females have faster decline rate of eGFR.

The average eGFR decreased across age groups, but different equations have different performances. Values were presented as mean and SD.

eGFR estimated glomerular filtration rate by CKD-EPI, FAS and Xiangya respectively.

\section{Differences between equations stratified by Scr level}

Partial correlation analysis of the difference between three equations and important related factors showed $\Delta$ (CKD-EPI, FAS), $\Delta$ (CKD-EPI, Xiangya) and $\Delta$ (FAS, Xiangya) were all significantly correlated with Scr and age. We plotted scatter diagrams to show the difference between these equations under different Scr level (Fig. 3). $\Delta$ (CKD-EPI, FAS) reduced with increasing Scr level when Scr was less than 0.9 mg/dL in males or $0.7 \mathrm{mg} / \mathrm{dL}$ in females, while was relatively stable when Scr levels more than above cut-off point. $\Delta$ (CKD-EPI, Xiangya) and $\Delta$ (FAS, Xiangya) were considerable with elevating Scr level. We performed agreement analysis by adopting $0.9 \mathrm{mg} / \mathrm{dL}$ in $\mathrm{males}$ and $0.7 \mathrm{mg} / \mathrm{dL}$ in females as the cut-off point, a substantial agreement between CKD-EPI and FAS equations was seen with Scr $\geq 0.9 \mathrm{mg} / \mathrm{dL}$ in $\mathrm{males}(\kappa 0.798)$ and Scr $\geq$ $0.7 \mathrm{mg} / \mathrm{dL}$ in females $(\kappa 0.745)$. Whereas agreement between Xiangya and other two equations were all slight $(\kappa<0.2)$. Agreement between equations was poor for subjects with Scr $<0.9 \mathrm{mg} / \mathrm{dL}$ in males and Scr $<0.7 \mathrm{mg} / \mathrm{dL}$ in females (Table S2 and Table S3).

The black, orange and blue circle represents the total, females and males, respectively. The solid line in the scatter plot represents the trend line. CKD-EPI: chronic kidney disease epidemiology collaboration; FAS full age spectrum; $\Delta$ : difference. 


\section{Differences between equations stratified by age}

As shown in Fig. 4, $\Delta$ (CKD-EPI, FAS), $\Delta$ (CKD-EPI, Xiangya) and $\Delta$ (FAS, Xiangya) existed differences across age groups. $\Delta$ (CKD-EPI, FAS) was relatively stable with aging. $\Delta$ (CKD-EPI, Xiangya) and $\Delta$ (FAS, Xiangya) have marked difference in females and males. In addition, we divided into six age groups, and used weighted $k$ statistic to quantify agreement between equations. We observed moderate to near-perfect in age 18-59 years ( $\kappa$ $0.617-0.901$ for males and $0.536-0.861$ for females) between CKD-EPI and FAS equations, whereas fair to moderate in age $\geq 60$ years ( $\kappa$ 0.494-0.513 for males and $0.373-0.526$ for females). However, the agreement in every age group was no more than moderate between Xiangya and CKD-EPI equations or FAS equations. In addition, CKD-EPI equation had higher estimates than FAS and Xiangya equations in GFR categories (Table S4 and Table S5).

The black, orange and blue circle represents the total, females and males, respectively. The solid line in the scatter plot represents the trend line. CKD-EPI: chronic kidney disease epidemiology collaboration; FAS full age spectrum; $\Delta$ : difference.

\section{Discussion}

In this study, we investigated the variety tendency of eGFR with aging using CKD-EPI, FAS and Xiangya equations and analyze the difference between these equations in healthy population.

eGFR decreased with aging, but the declining tendency of it based on different equations was diverse. eGFR by CKD-EPI and Xiangya equations declined gradually from 18 years with a rate of $0.81 \mathrm{~mL} / \mathrm{min} / 1.73 \mathrm{~m}^{2} /$ year and $0.56 \mathrm{~mL} / \mathrm{min} / 1.73 \mathrm{~m}^{2} /$ year, respectively. Meanwhile, eGFR by FAS equations declined at a rate of $1.27 \mathrm{~mL} / \mathrm{min} / 1.73 \mathrm{~m}^{2} /$ year after 40 years old, which was similar with the result of previous studies based on mGFR. A study by Ma YC et al. including 301 Chinese healthy subjects ( 130 males and 171 females), revealed that mGFR of adults (18-50 years) in males and females were approximately $104.0 \mathrm{~mL} / \mathrm{min} / 1.73 \mathrm{~m}^{2}$ and $110.1 \mathrm{~mL} / \mathrm{min} / 1.73 \mathrm{~m}^{2}$, and then declined at a rate of $12.2 \mathrm{~mL} / \mathrm{min} / 1.73$ $\mathrm{m}^{2} /$ decade[14]. In 2017, Denic et al. found the number of nephrons per kidney and the total mGFR declined with aging, especially above 40 years old based on healthy adult kidney donors[15]. Besides, a meta-analysis by Pottel et al. also supported the construction of 40 years old as the descent inflection point of declining GFR with aging[16]. The eGFR by Xiangya was lower than these by other equations and mGFR in previous studies. Though the development and validation group of Xiangya equation were Chinese, $30.81 \%$ of these were patients with CKD and sample size was relatively few, which may result in the quite differences between Xiangya and other estimated equations and mGFR in healthy people. Thus, we mainly compared the performance of CKD-EPI and FAS equation in evaluating eGFR in this study. Therefore, FAS equation may be more suitable to evaluate annual rate of decline compared with other equations in healthy subjects.

Compared with males, the female had a higher eGFR level after adulthood and a faster decrease of it with aging. Xu $\mathrm{R}$ et al. also reported the difference of declining rate in eGFR with elevating age between healthy female and male groups $\left(0.51 \mathrm{~mL} / \mathrm{min} / 1.73 \mathrm{~m}^{2} /\right.$ year in males vs. 0.74 $\mathrm{mL} / \mathrm{min} / 1.73 \mathrm{~m}^{2} /$ year in females) [17]. On the contrary, another study that used clearances of inulin described a slower fall in GFR with age in females [18]. Some previous studies have revealed the protective role of osetragen in kidney function [19], which contribute to the higher eGFR level in females under 50 years. In the term of declining rate of eGFR, one possible explanation is that kidney function is preserved as healthy subjects age at the expense of a gradual decrease in the kidney functional reserve [20]. Men may have a higher reserved kidney function to compensate for the accumulation of sclerotic glomeruli with aging, which results in the slower declining rate of eGFR in males.

In our scatter plot related to creatinine, we observed the considerable differences between three equations with Scr levels less than $0.7 \mathrm{mg} / \mathrm{dL}$ in males and $0.9 \mathrm{mg} / \mathrm{dL}$ in females, and agreement between equations was poor. The cut-off point may be the result of Scr knot value in the development of CKD-EPI equation. Studies have shown the accuracy of FAS equation in evaluating GFR is poorer at low Scr values than that at high Scr levels[6]. A new European Kidney Function Consortium (EKFC) equation was designed to addresses this issue, and may be more accurate in subjects with low creatinine level [21]. We also tested the consistency between EKFC and other equations, and observed that the consistency between EKFC and CKD-EPI equations was better than that between CKD-EPI and FAS equations. However, the accuracy in evaluating eGFR of EKFC was undefined and need more verification. Thus, further research is needed to evaluate the eGFR estimating equations in subjects with low Scr value. When Scr levels more than above cut-off point, $\triangle$ (CKD-EPI, FAS) was relatively small and stable. We quantified agreement among the three equations, the result showed greater agreement between CKD-EPI and FAS equation with Scr $\geq 0.7$ in females and Scr $\geq 0.9$ $\mathrm{mg} / \mathrm{dL}$ in males. Therefore, according to creatinine level, we tend to suggest the application of the FAS or CKD-EPI equation to evaluate eGFR in healthy females with $\mathrm{Scr} \geq 0.7$ and healthy males with $\mathrm{Scr} \geq 0.9 \mathrm{mg} / \mathrm{dL}$.

In our scatter plot related to age, $\triangle(C K D$-EPI, FAS) was relatively stable with aging. Agreement analysis showed moderate to near-perfect agreement between CKD-EPI and FAS equations in non-elderly subjects, but worse agreement between these equations in older adults. The agreement of CKD-EPI and FAS equation was slightly better in males compared with this in females. In older population, most of the eGFR values calculated by CKD-EPI equation were higher than that by FAS equation. A Meta-Analysis by Eriksen et al. [22] distributed a slightly higher eGFR by CKD-EPI equation than mGFR both males and females. We also performed agreement analysis among subgroups according to age and Scr level, which is consistent with above results. The agreement was slight between Xiangya equation and CKD-EPI or FAS equation. Therefore, the clinicial 
may consider CKD-EPI or FAS equation to estimate eGFR in non-elderly population, yet FAS equation could be more suitable for healthy older adults. Besides, clinicians may need to be cautious when using Xiangya equation.

Compared with previous studies, the advantages of this study were as follows: (1) A large number of healthy subjects were included, thus the variation of eGFR with aging in this study could be a reference to clinician when evaluating kidney function; (2) We firstly analyse the difference and agreement between CKD-EPI, FAS and Xiangya equations in Chinese healthy population, and make recommendations according to the results. However, the limitation of this study is that there is no mGFR to analyse variety tendency of GFR with aging and evaluate the accuracy of these equations. To carry out the mGFR examination items wildly is difficult in healthy individuals, so we adopt estimated equations to calculate eGFR in this large sample population. Another limitation is that this research is a retrospective cross-sectional study.

\section{Conclusions}

FAS equation could be used to evaluate declining rate of eGFR in healthy population. We recommend FAS or CKD-EPI equation in non-elderly adults, and FAS equation in older adults to calculate eGFR. The clinicians may need to be cautious when using Xiangya equation in healthy Chinese adults. More verification is needed, especially in older adults and population with low Scr level.

\section{Abbreviations}

Scr: serum creatinine; GFR: glomerular filtration rate; AKI: acute kidney injury; KDIGO:Kidney Disease Improving Global Outcomes; CKD-EPI: chronic kidney disease epidemiology collaboration; FAS: full age spectrum; BMI: body mass index; BP: blood pressure; FBG: fasting blood glucose; UA: uric acid; ALT: alanine aminotransferase; HDL-C: high density lipoprotein cholesterol; LDL-C: low-density lipoprotein cholesterol; TC: total cholesterol; FBG: fasting blood glucose; SD: standard deviation; $\Delta$ : difference; EKFC: European Kidney Function Consortium; mGFR: measured glomerular filtration rate

\section{Declarations}

\section{Ethics approval and consent to participate}

This study was approved by the ethical review committee at the First Affiliated Hospital of Nanjing medical University (2018-SR-181).

\section{Consent for publication}

Not applicable

\section{Availability of data and materials}

The datasets used and analysed during the current study are available from the corresponding author on reasonable request.

\section{Competing interests}

The authors declare that they have no competing interests.

\section{Funding}

This work was supported by National Natural Science Foundation of China (H2501-81971263), Jiangsu Provincial Key Discipline of Medicine (ZDXKA2016003), Jiangsu Province Medical Talents Program (ZDRCA2016021), Jiangsu Province Cadres Health Care Project (BJ17018), and Jiangsu Province Hospital 511 Project (JSPH-511A-2018-5).

\section{Authors' contributions}

Conception and design, acquisition of data, analysis and interpretation of data, drafting and revising the article, and final approval of the version to be published: WL, SX. Revising the article for important intellectual content and final approval of the version to be published: YZZ, ZJ.

Acquisition of data and final approval of the version to be published: ZQ. Conception and design, acquisition of data, analysis and interpretation of data, revising the article for important intellectual content, and final approval of the version to be published: $\mathrm{ZWH}$.

\section{Acknowledgements}

The authors acknowledge the efforts of Huang Peng for his guidance on statistics.

\section{References}


1. Denic A, Glassock RJ, Rule AD. Structural and Functional Changes With the Aging Kidney. Adv Chronic Kidney Dis. 2016 Jan;23(1):19-28.

2. Schmitt R, Cantley LG. The impact of aging on kidney repair. Am J Physiol Renal Physiol. 2008 Jun;294(6):F1265-72.

3. Zhang L, Wang F, Wang L, Wang W, Liu B, Liu J, Chen M, He Q, Liao Y, Yu X, Chen N, Zhang JE, Hu Z, Liu F, Hong D, Ma L, Liu H, Zhou X, Chen J, Pan L, Chen W, Wang W, Li X, Wang H. Prevalence of chronic kidney disease in China: a cross-sectional survey. Lancet. 2012 Mar 3;379(9818):815 - 22.

4. Stevens LA, Coresh J, Greene T, Levey AS. Assessing kidney function-measured and estimated glomerular filtration rate. N Engl J Med. 2006 Jun 8;354(23):2473-83.

5. Shemesh O, Golbetz H, Kriss JP, Myers BD. Limitations of creatinine as a filtration marker in glomerulopathic patients. Kidney Int. 1985 Nov;28(5):830-8.

6. Pottel H, Hoste L, Dubourg L, Ebert N, Schaeffner E, Eriksen BO, Melsom T, Lamb EJ, Rule AD, Turner ST, Glassock RJ, De Souza V, Selistre L, Mariat C, Martens F, Delanaye P. An estimated glomerular filtration rate equation for the full age spectrum. Nephrol Dial Transplant. 2016 May;31(5):798-806.

7. Li DY, Yin WJ, Yi YH, Zhang BK, Zhao J, Zhu CN, Ma RR, Zhou LY, Xie YL, Wang JL, Zuo SR, Liu K, Hu C, Zhou G, Zuo XC. Development and validation of a more accurate estimating equation for glomerular filtration rate in a Chinese population. Kidney Int. 2019 Mar;95(3):636-646.

8. Levey AS, Stevens LA, Schmid CH, Zhang YL, Castro AF 3rd, Feldman HI, Kusek JW, Eggers P, Van Lente F, Greene T, Coresh J; CKD-EPI (Chronic Kidney Disease Epidemiology Collaboration). A new equation to estimate glomerular filtration rate. Ann Intern Med. 2009 May 5;150(9):604-12.

9. Matsuo S, Imai E, Horio M, Yasuda Y, Tomita K, Nitta K, Yamagata K, Tomino Y, Yokoyama H, Hishida A; Collaborators developing the Japanese equation for estimated GFR. Revised equations for estimated GFR from serum creatinine in Japan. Am J Kidney Dis. 2009 Jun;53(6):982-92.

10. Liao Y, Liao W, Liu J, Xu G, Zeng R. Assessment of the CKD-EPI equation to estimate glomerular filtration rate in adults from a Chinese CKD population. J Int Med Res. 2011;39(6):2273-80.

11. Zhou LY, Yin WJ, Zhao J, Zhang BK, Hu C, Liu K, Wang JL, Zhou G, Chen LH, Zuo SR, Xie YL, Zuo XC. A Novel Creatinine-Based Equation to Estimate Glomerular Filtration Rate in Chinese Population With Chronic Kidney Disease: Implications for DOACs Dosing in Atrial Fibrillation Patients. Front Pharmacol. 2021 Feb 19;12:615953.

12. Gao JQ, Zhao FG, Huang JM, Shao FQ, Xie P. Comparative performance of FAS equation and Asian modified CKD-EPI in the determination of GFR in Chinese patients with CKD with the 99mTc-DTPA plasma clearance as the reference method. Nefrologia. 2021 Jan-Feb;41(1):27-33.

13. Yong Z, Li F, Pei X, Liu X, Song D, Zhang X, Zhao W. A comparison between 2017 FAS and 2012 CKD-EPI equations: a multi-center validation study in Chinese adult population. Int Urol Nephrol. 2019 Jan;51(1):139-146.

14. Ma YC, Zuo L, Chen L, Su ZM, Meng S, Li JJ, Zhang CL, Wang HY. Distribution of measured GFR in apparently healthy Chinese adults. Am J Kidney Dis. 2010 Aug;56(2):420-1.

15. Denic A, Mathew J, Lerman LO, Lieske JC, Larson JJ, Alexander MP, Poggio E, Glassock RJ, Rule AD. Single-Nephron Glomerular Filtration Rate in Healthy Adults. N Engl J Med. 2017 Jun 15;376(24):2349-2357.

16. Pottel H, Hoste L, Yayo E, Delanaye P. Glomerular Filtration Rate in Healthy Living Potential Kidney Donors: A Meta-Analysis Supporting the Construction of the Full Age Spectrum Equation. Nephron. 2017;135(2):105-119.

17. Xu R, Zhang LX, Zhang PH, Wang F, Zuo L, Wang HY. Gender differences in age-related decline in glomerular filtration rates in healthy people and chronic kidney disease patients. BMC Nephrol. 2010 Aug 23;11:20.

18. Berg UB. Differences in decline in GFR with age between males and females. Reference data on clearances of inulin and PAH in potential kidney donors. Nephrol Dial Transplant. 2006 Sep;21(9):2577-82.

19. Baylis C. Changes in renal hemodynamics and structure in the aging kidney; sexual dimorphism and the nitric oxide system. Exp Gerontol. 2005 Apr;40(4):271-8.

20. Fuiano G, Sund S, Mazza G, Rosa M, Caglioti A, Gallo G, Natale G, Andreucci M, Memoli B, De Nicola L, Conte G. Renal hemodynamic response to maximal vasodilating stimulus in healthy older subjects. Kidney Int. 2001 Mar;59(3):1052-8.

21. Pottel H, Björk J, Courbebaisse M, Couzi L, Ebert N, Eriksen BO, Dalton RN, Dubourg L, Gaillard F, Garrouste C, Grubb A, Jacquemont L, Hansson M, Kamar N, Lamb EJ, Legendre C, Littmann K, Mariat C, Melsom T, Rostaing L, Rule AD, Schaeffner E, Sundin PO, Turner S, Bökenkamp A, Berg U, Åsling-Monemi K, Selistre L, Åkesson A, Larsson A, Nyman U, Delanaye P. Development and Validation of a Modified Full Age Spectrum Creatinine-Based Equation to Estimate Glomerular Filtration Rate: A Cross-sectional Analysis of Pooled Data. Ann Intern Med. 2021 Feb;174(2):183-191.

22. Eriksen BO, Palsson R, Ebert N, Melsom T, van der Giet M, Gudnason V, Indridason OS, Inker LA, Jenssen TG, Levey AS, Solbu MD, Tighiouart $\mathrm{H}$, Schaeffner E. GFR in Healthy Aging: an Individual Participant Data Meta-Analysis of lohexol Clearance in European Population-Based Cohorts. J Am Soc Nephrol. 2020 Jul;31(7):1602-1615.

Page $7 / 11$ 
Figures

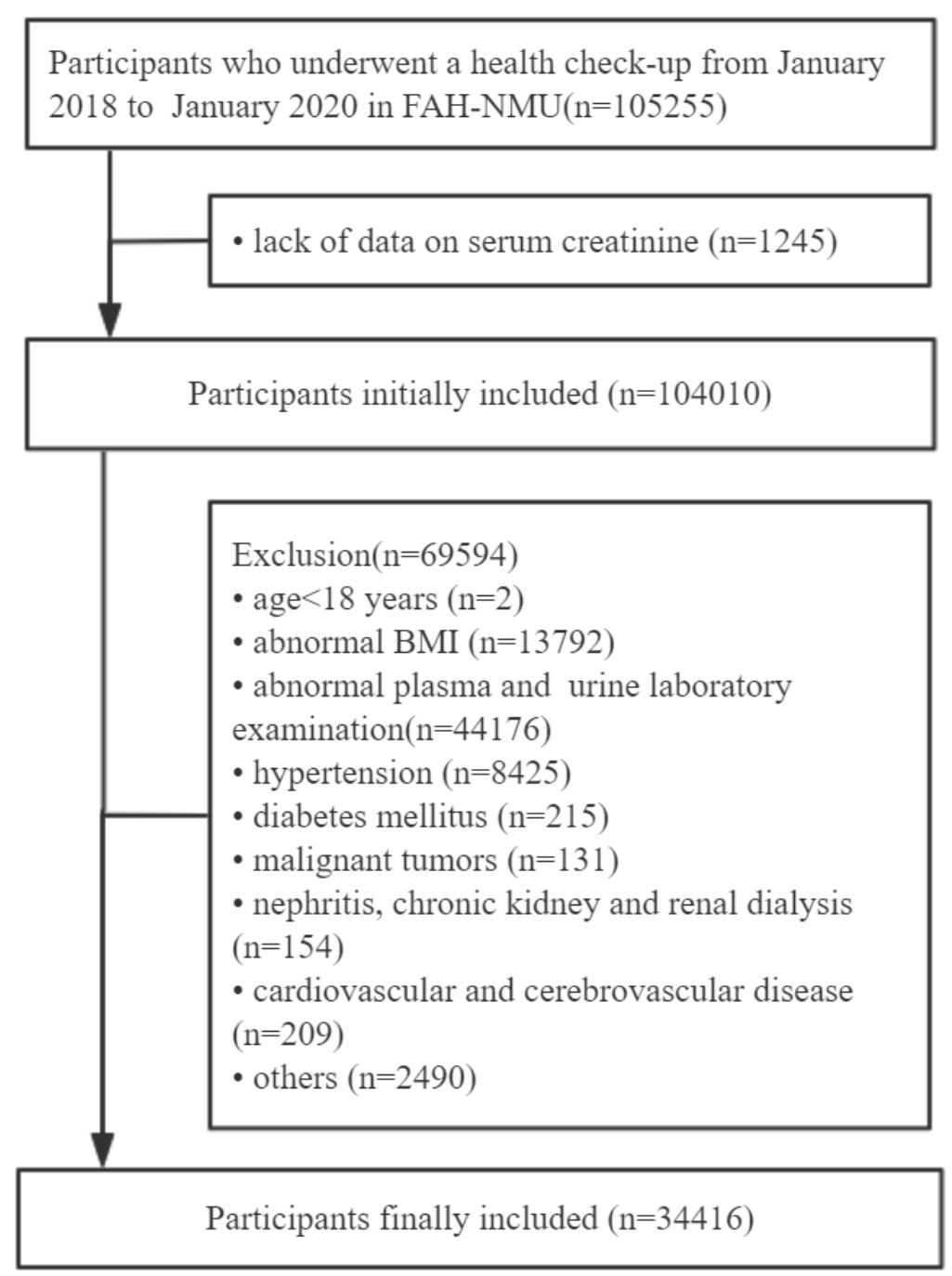

Figure 1

The screening flowchart for the eligible participates. 
Males

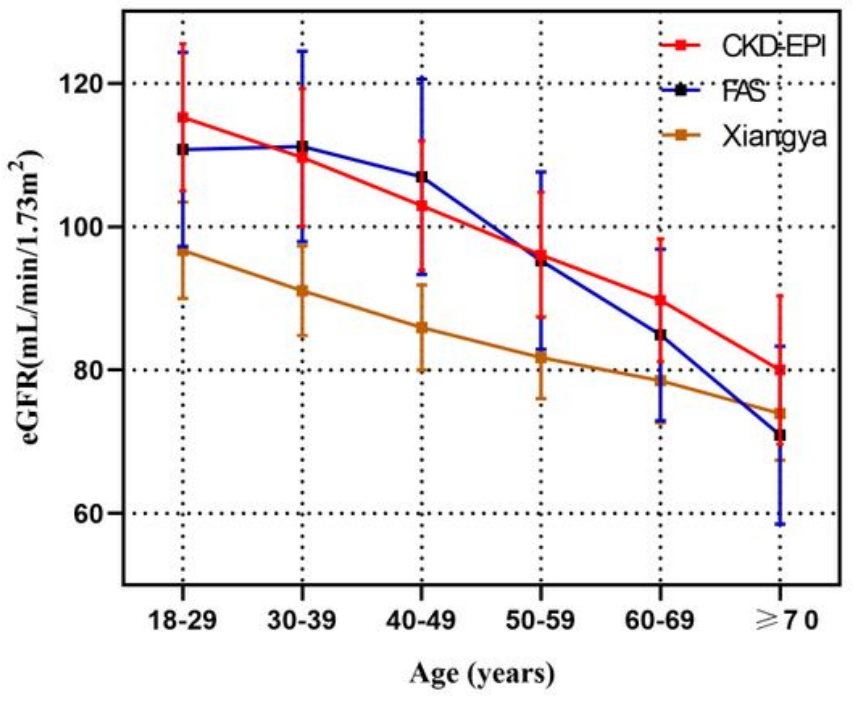

a

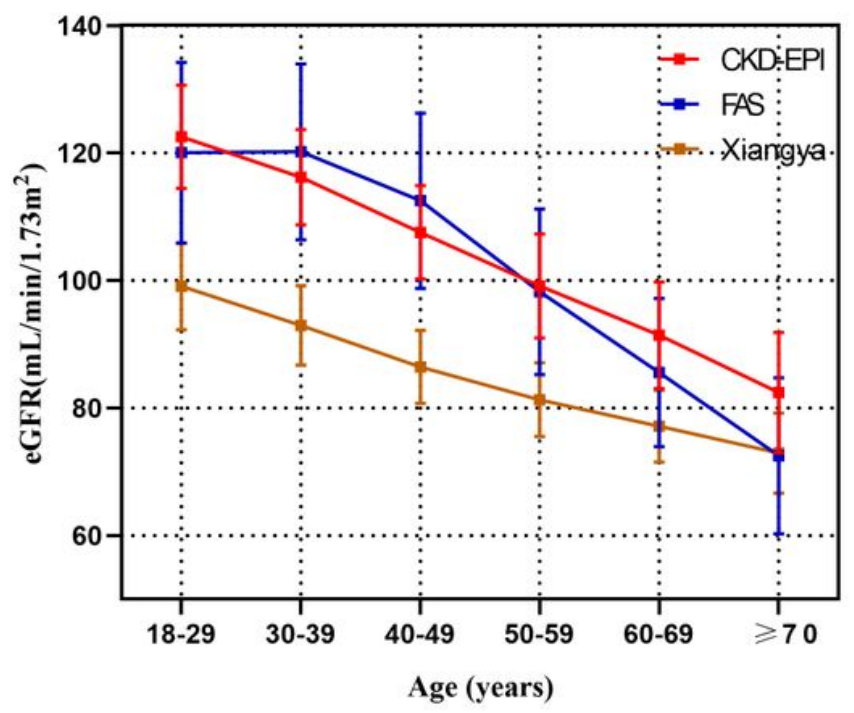

b

Figure 2

Mean eGFR across age groups in healthy population. 

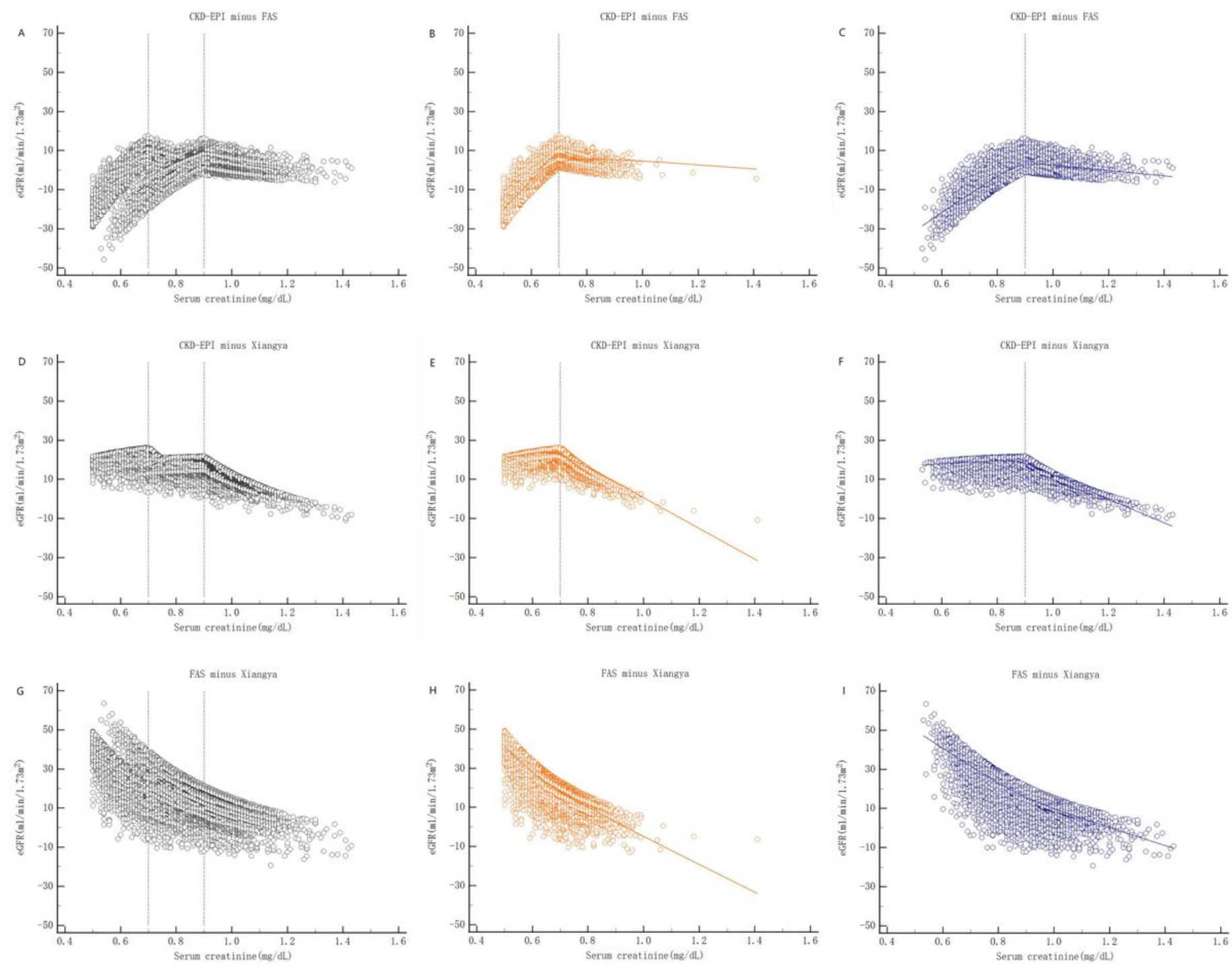

Figure 3

Scatter plot of $\Delta($ CKD-EPI, FAS $), \Delta($ CKD-EPI, Xiangya) and $\Delta$ (FAS, Xiangya) correlated to Scr. 

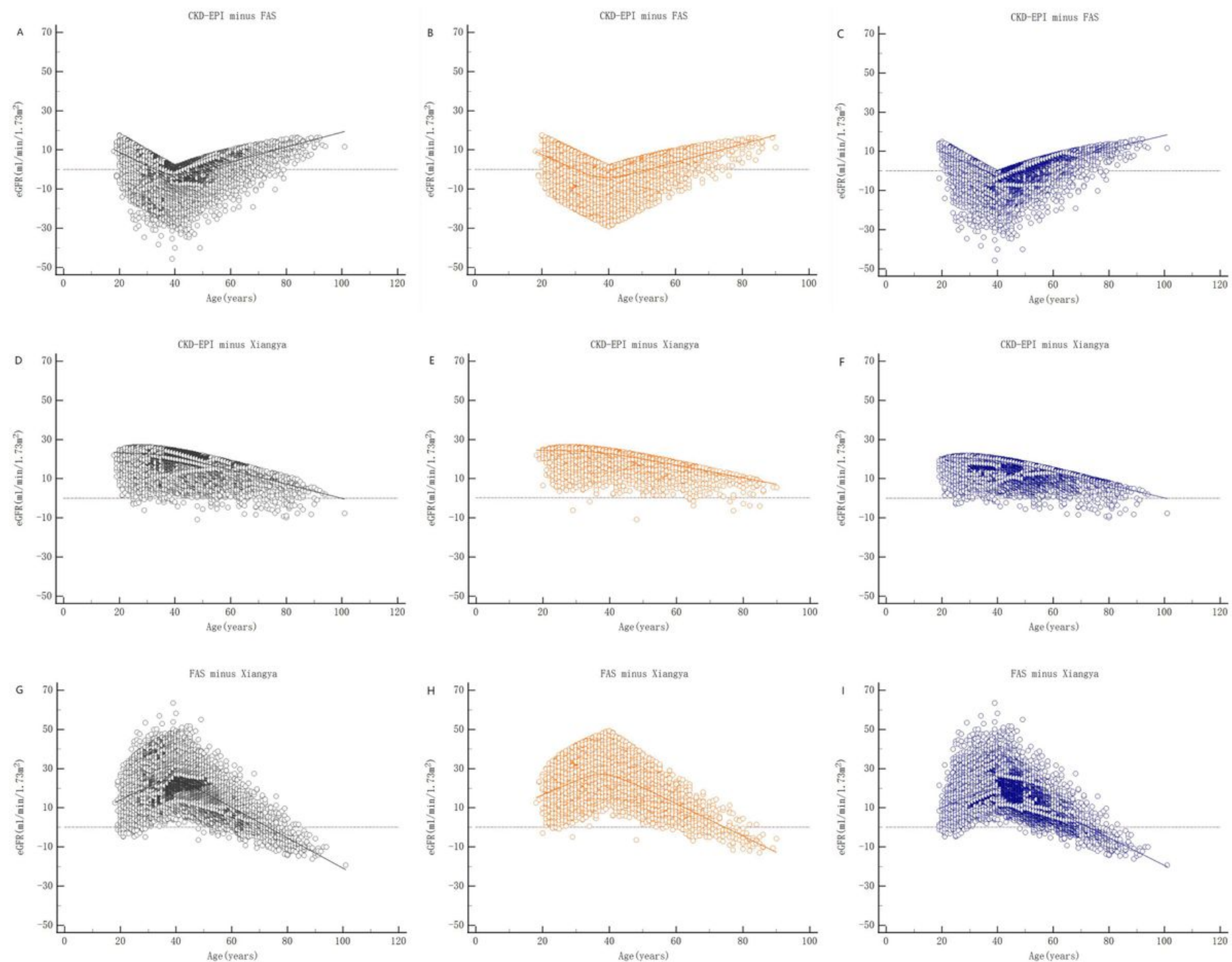

Figure 4

Scatter plot of $\Delta$ (CKD-EPI, FAS), $\Delta($ CKD-EPI, Xiangya) and $\Delta$ (FAS, Xiangya) correlated to age.

\section{Supplementary Files}

This is a list of supplementary files associated with this preprint. Click to download.

- Tablesupplement13.docx

- Tables4.xlsx

- TableS5.xlsx 